\title{
One-Dimensional Time-Dependent Ising Model in an External Magnetic Field
}

\author{
M. BŁASZYK \\ Institute of Physics, A. Mickiewicz University \\ Umultowska 85, 61-614 Poznań, Poland
}

(Received May 19, 2008)

\begin{abstract}
The relaxation process, through which the inequilibrium state described by the Ising model reaches the equilibrium state, was proposed. The process is defined by the action of the transition matrix on the vector of state and the demand of its normalisation. It is assumed that in the state of inequilibrium the vector of state has the same functional dependence as the eigenvector of the transition matrix corresponding to the highest eigenvalue. Two cases are considered: when the cell under transformation is composed of one or two sites. The calculations were performed for a uniform initial state. For the two cases of one and two-site cells the modes of reaching the equilibrium state via magnetisation were compared. When the external magnetic field and temperature tend to zero, both magnetisation and the correlation function of the nearest neighbours show the critical slowing down phenomenon.
\end{abstract}

PACS numbers: $05.50 .+\mathrm{q}$

\section{Introduction}

Investigation of the phenomena taking place outside the state of thermodynamical equilibrium is equally interesting and imprecise at least for the Hamiltonians devoid of natural dynamics. A typical example of such Hamiltonians is the Ising model. The need to establish its behaviour outside the equilibrium state has forced formulation of the kinetic versions of this model [1]. Because it is believed that the Ising model does not have internal dynamics, it is introduced on the basis of the master equation. The choice of the transition probabilities permits determination of the process of reaching the equilibrium state. By this choice it is possible to define the Glauber model in which the magnetisation tends to the equilibrium state and the Kawasaki model in which the magnetisation is constant and the process of reaching equilibrium is that of reaching the homogeneous distribution of the sites with the positive and negative magnetic moments. However, always the dynamics is introduced by the master equation, which can be justified only for the states close to that of the equilibrium.

In another approach the dynamics is based on the cellular automata, whose evolution is described by the a priori rules [2]. In this approach it is possible to relate the behaviour of the $d$-dimensional cellular automata with that of the $d+1$ equilibrium Ising model [3]. The disadvantage of this approach is that only a single microstate and not a whole ensemble can be analysed. Therefore, although it is possible to trace the evolution of one state it is difficult to describe the evolution of the system with the specific interactions for the preset initial conditions.

The kinetic Ising model proposed below is intermediate between these two approaches and in some aspects improves both of them. Relative to the widely used dynamics based on the master equation (the Glauber and Kawasaki dynamics) it does not require the use of this equation. Moreover, as it can describe only the infinite systems, it removes the problems related to the Bogoliubov-Born-Green-Kirkwood-Yvon (BBGKY) hierarchy [4], i.e. the necessity of solving all $2^{N}$ equations for the correlation functions. The model proposed has the advantages of the Glauber approach, i.e. it leads the system to the state of thermal equilibrium and shows the critical slowing down near the critical point.

In comparison to the cellular automata approach, the model we propose uses the language of probabilities, so it can describe the whole ensemble of systems and is not limited to following the evolution of a single microstate. The disadvantages of our model are related to the choice of the initial conditions. They cannot be arbitrary as in the Glauber or Kawasaki dynamics. However, it is relatively easy to describe the evolution of a system if the initial inequilibrium state is identical to the equilibrium state for variable conditions.

\section{The system and its dynamics}

We are concerned here with the behaviour of the expected values of the magnetic moments $\left\langle S_{i}\right\rangle(t)$. We will assume that for the model with the nearest neighbour interactions the magnetic moment value at time $t$ at the $i$-th site is fully described by the magnetic moments of neighbouring sites: $(i-1)$-th and $(i+1)$-th at the time $t-1$. The relationship is shown in Fig. 1 .

Let us assume that at time $t=0$, the probability that the $i$-th spin is directed upwards is defined, or equivalently their magnetisation at each site of the system is 


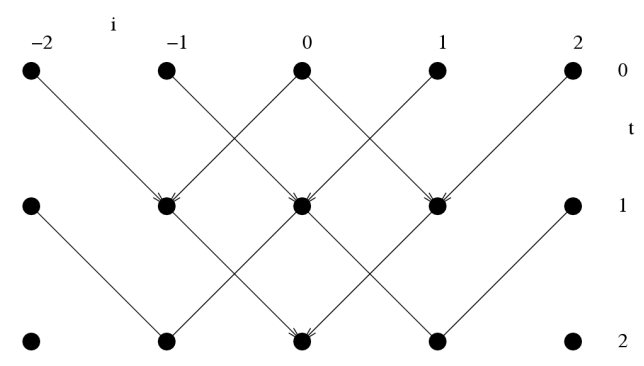

Fig. 1. Two-dimensional spin lattice, $i$ stands for the space dimension, $t$ stands for time. The arrows indicate the spin state exerting the effect on a given site.

defined. The interaction between the sites is described by the Hamiltonian

$$
\mathcal{H}=-J \sum_{i} S_{i} S_{j}-H \sum_{i} S_{i}
$$

with $S_{i}= \pm 1$. The corresponding transition matrix is

$$
\boldsymbol{M}=\left[\begin{array}{cc}
\mathrm{e}^{K+B} & \mathrm{e}^{-K} \\
\mathrm{e}^{-K} & \mathrm{e}^{K-B}
\end{array}\right],
$$

where $K=\beta J, B=\beta H$ and $\beta=1 / k T$. It has been shown in [5] that the equilibrium state can be described in a way similar to that used in quantum mechanics, with the help of the bra and ket vectors such that

$$
\boldsymbol{M}\left|\psi_{+}\right\rangle=\lambda_{+}\left|\psi_{+}\right\rangle, \quad\left\langle\psi_{+}\right| \boldsymbol{M}=\lambda_{+}\left\langle\psi_{+}\right|,
$$

where $\lambda_{+}=\mathrm{e}^{K} \cosh B+\mathrm{e}^{K} \sqrt{\sinh ^{2} B+\mathrm{e}^{-4 K}}$. The normalisation condition imposed on the vectors $\left\langle\psi_{+} \mid \psi_{+}\right\rangle=1$ means that the first coordinates of the vectors can be written as $\left\langle\psi_{+}|[1]=| \psi_{+}\right\rangle[1]=\sqrt{\frac{1}{2}(1+\langle S\rangle)}$, while the second coordinates as $\left\langle\psi_{+}|[2]=| \psi_{+}\right\rangle[2]=$ $\sqrt{\frac{1}{2}(1-\langle S\rangle)}$, where $\langle S\rangle$ is the magnetisation and $\langle S\rangle=$ $\sinh B / \sqrt{\sinh ^{2} B+\mathrm{e}^{-4 K}}$. The functional expressions describing these coordinates follow from the demand that the product of the vector coordinates bra and ket would give the probabilities of finding the system in a corresponding state, so $\left\langle\psi_{+}|[1] \cdot| \psi_{+}\right\rangle[1]=P(\uparrow)=\frac{1}{2}(1+\langle s\rangle)$, $\left\langle\psi_{+}|[2] \cdot| \psi_{+}\right\rangle[2]=P(\downarrow)=\frac{1}{2}(1-\langle s\rangle)$, where $P(\uparrow)$ and $P(\downarrow)$ is the probability of the spin directed upwards or downwards. Similar relations are found for a smaller eigenvalue $\lambda_{-}=\mathrm{e}^{K} \cosh B-\mathrm{e}^{K} \sqrt{\sinh ^{2} B+\mathrm{e}^{-4 K}}$ :

$$
\boldsymbol{M}\left|\psi_{-}\right\rangle=\lambda_{-}\left|\psi_{-}\right\rangle, \quad\left\langle\psi_{-}\right| \boldsymbol{M}=\lambda_{-}\left\langle\psi_{-}\right| .
$$

Again the normalisation condition implies the functional expressions of the bra and ket vectors, however, now the states are exchanged and the first coordinate corresponds to $P(\downarrow)$ and the second to $P(\uparrow)$. Therefore, we can write $\left\langle\psi_{-}|[1]=| \psi_{-}\right\rangle[1]=\sqrt{\frac{1}{2}(1-\langle s\rangle)}$ and $\left\langle\psi_{-}|[2]=| \psi_{-}\right\rangle[2]=-\sqrt{\frac{1}{2}(1+\langle s\rangle)}$, where the minus sign is the phase factor ensuring the orthogonality of the states.

This description of the equilibrium state can be applied to the inequilibrium states. Let us assume that the single site state (inequilibrium) is also described by the state vector $|\phi\rangle$ and that both coordinates of the state satisfy the same functional dependences as those met by the coordinates of the state $\left|\psi_{+}\right\rangle$in the equilibrium state. No other assumptions are taken as to the vector $|\phi\rangle$, in particular on the difference between the vector and the equilibrium state. On the basis of the completeness of the space spanned by the vectors $\left|\psi_{+}\right\rangle$and $\left|\psi_{-}\right\rangle,|\phi\rangle$ can be written as

$$
|\phi\rangle=\left\langle\psi_{+} \mid \phi\right\rangle\left|\psi_{+}\right\rangle+\left\langle\psi_{-} \mid \phi\right\rangle\left|\psi_{-}\right\rangle .
$$

If the vector $|\phi\rangle$ describes the state of the $i$-th site at time $t=0$, then this state will affect the behaviour of the neighbouring sites $(i-1)$-th and $(i+1)$-th at $t=1$. These effects can be described by the expressions describing the vectors $\boldsymbol{M}|\phi\rangle$ and $\langle\phi| \boldsymbol{M}$. To be able to relate these vectors with the probability, we have to normalise them each time after multiplication by matrix $\boldsymbol{M}$. The probability of the $i$-th spin to be directed upwards is the arithmetic mean of the probabilities of the effects coming from the $(i-1)$-th and $(i+1)$-th site. The above procedure becomes so complex that for an arbitrary initial state it becomes practically unworkable. The only case simple enough to be studied is the homogeneous state, when for each site $i$ the magnetic moment $\left\langle S_{i}\right\rangle(t)$ takes the same initial value $\langle S\rangle\left(t_{0}\right)$. The procedure maintains the homogeneity of the system so the system will be described by only one variable $\langle S\rangle(t)$, which will depend on time but will not change from site to site. The normalised state $\left|\phi_{1}\right\rangle$ obtained as a result of the action of matrix $\boldsymbol{M}$ on the state (Eq. (5)) at zero time $\left|\phi_{1}\right\rangle$ can be expressed as

$$
\left|\phi_{1}\right\rangle=C_{1} \boldsymbol{M}\left|\phi_{0}\right\rangle
$$

where $C_{1}$ is the normalising constant. On the basis of Eqs. (3)-(5), Eq. (6) can be rewritten as

$$
\begin{aligned}
& \left|\phi_{1}\right\rangle= \\
& \frac{\lambda_{+}\left\langle\psi_{+} \mid \phi_{0}\right\rangle\left|\psi_{+}\right\rangle+\lambda_{-}\left\langle\psi_{-} \mid \phi_{0}\right\rangle\left|\psi_{-}\right\rangle}{\sqrt{\lambda_{+}^{2}\left\langle\psi_{+} \mid \phi_{0}\right\rangle\left\langle\phi_{0} \mid \psi_{+}\right\rangle+\lambda_{-}^{2}\left\langle\psi_{-} \mid \phi_{0}\right\rangle\left\langle\phi_{0} \mid \psi_{-}\right\rangle}}
\end{aligned}
$$

Subjecting the state $\left|\phi_{0}\right\rangle n$-times to matrix $\boldsymbol{M}$, we get $\left|\phi_{n}\right\rangle$ in the form

$$
\begin{aligned}
& \left|\phi_{n}\right\rangle= \\
& \frac{\lambda_{+}^{n}\left\langle\psi_{+} \mid \phi_{0}\right\rangle\left|\psi_{+}\right\rangle+\lambda_{-}^{n}\left\langle\psi_{-} \mid \phi_{0}\right\rangle\left|\psi_{-}\right\rangle}{\sqrt{\lambda_{+}^{2 n}\left\langle\psi_{+} \mid \phi_{0}\right\rangle\left\langle\phi_{0} \mid \psi_{+}\right\rangle+\lambda_{-}^{2 n}\left\langle\psi_{-} \mid \phi_{0}\right\rangle\left\langle\phi_{0} \mid \psi_{-}\right\rangle}} .
\end{aligned}
$$

Since $\left\langle\phi_{n}|[1] \cdot| \phi_{n}\right\rangle[1]=P_{n}(\uparrow)$ and $\left\langle\phi_{n}|[2] \cdot| \phi_{n}\right\rangle[2]=P_{n}(\downarrow)$ the probabilities of the spin being directed upwards and downwards at time $n$ can be written as 


$$
\begin{aligned}
& P_{n}(\uparrow)=\frac{\lambda_{+}^{2 n}\left(\left\langle\psi_{+} \mid \phi_{0}\right\rangle\right)^{2} \frac{1}{2}(1+\langle S\rangle)+\lambda_{-}^{2 n}\left(\left\langle\psi_{-} \mid \phi_{0}\right\rangle\right)^{2} \frac{1}{2}(1-\langle S\rangle)+\lambda_{+}^{n} \lambda_{-}^{n}\left\langle\psi_{+} \mid \phi_{0}\right\rangle\left\langle\phi_{0} \mid \psi_{-}\right\rangle \sqrt{1-\langle S\rangle^{2}}}{\lambda_{+}^{2 n}\left(\left\langle\psi_{+} \mid \phi_{0}\right\rangle\right)^{2}+\lambda_{-}^{2 n}\left(\left\langle\psi_{-} \mid \phi_{0}\right\rangle\right)^{2}}, \\
& P_{n}(\downarrow)=\frac{\lambda_{+}^{2 n}\left(\left\langle\psi_{+} \mid \phi_{0}\right\rangle\right)^{2} \frac{1}{2}(1-\langle S\rangle)+\lambda_{-}^{2 n}\left(\left\langle\psi_{-} \mid \phi_{0}\right\rangle\right)^{2} \frac{1}{2}(1+\langle S\rangle)-\lambda_{+}^{n} \lambda_{-}^{n}\left\langle\psi_{+} \mid \phi_{0}\right\rangle\left\langle\phi_{0} \mid \psi_{-}\right\rangle \sqrt{1-\langle S\rangle^{2}}}{\lambda_{+}^{2 n}\left(\left\langle\psi_{+} \mid \phi_{0}\right\rangle\right)^{2}+\lambda_{-}^{2 n}\left(\left\langle\psi_{-} \mid \phi_{0}\right\rangle\right)^{2}},
\end{aligned}
$$

where $\langle S\rangle$ is the equilibrium magnetisation, that is the magnetic moment which will be reached at $t \rightarrow \infty$. Equation (9) has been written taking advantage of the relations $\left\langle\phi_{0} \mid \psi_{+}\right\rangle=\left\langle\psi_{+} \mid \phi_{0}\right\rangle$ and $\left\langle\phi_{0} \mid \psi_{-}\right\rangle=\left\langle\psi_{-} \mid \phi_{0}\right\rangle$. In terms of these probabilities we can write the expression for the expected value of the spin at $t=n$ :

$$
\langle S\rangle_{n}=\frac{\left[\lambda_{+}^{2 n}\left(\left\langle\psi_{+} \mid \phi_{0}\right\rangle\right)^{2}-\lambda_{-}^{2 n}\left(\left\langle\psi_{-} \mid \phi_{0}\right\rangle\right)^{2}\right]\langle S\rangle+\lambda_{+}^{n} \lambda_{-}^{n}\left\langle\psi_{+} \mid \phi_{0}\right\rangle\left\langle\phi_{0} \mid \psi_{-}\right\rangle \sqrt{1-\langle S\rangle^{2}}}{\lambda_{+}^{2 n}\left(\left\langle\psi_{+} \mid \phi_{0}\right\rangle\right)^{2}+\lambda_{-}^{2 n}\left(\left\langle\psi_{-} \mid \phi_{0}\right\rangle\right)^{2}} .
$$

The time dependence is involved only through the power terms of the eigenvalues $\lambda_{+}$and $\lambda_{-}$. This expression can be rewritten as

$$
\langle S\rangle_{n}=\frac{\left[\left(\left\langle\psi_{+} \mid \phi_{0}\right\rangle\right)^{2}-\mathrm{e}^{2 n \ln \left(\lambda_{-} / \lambda_{+}\right)}\left(\left\langle\psi_{-} \mid \phi_{0}\right\rangle\right)^{2}\right]\langle S\rangle+2 \mathrm{e}^{n \ln \left(\lambda_{-} / \lambda_{+}\right)}\left\langle\psi_{+} \mid \phi_{0}\right\rangle\left\langle\phi_{0} \mid \psi_{-}\right\rangle \sqrt{1-\langle S\rangle^{2}}}{\left(\left\langle\psi_{+} \mid \phi_{0}\right\rangle\right)^{2}+\mathrm{e}^{2 n \ln \left(\lambda_{-} / \lambda_{+}\right)}\left(\left\langle\psi_{-} \mid \phi_{0}\right\rangle\right)^{2}} .
$$

Analysis of the numerator of Eq. (11) implies that the process of reaching equilibrium by the magnetisation is described by only two relaxation times $\tau_{1}=$ $\left[\ln \left(\lambda_{-} / \lambda_{+}\right)\right]^{-1}$ and $\tau_{2}=2 \tau_{1}$, however, it is not the case as the exponential function occurs also in one of the two terms of the denominator. Therefore, the above expression can be written as an infinite sum of terms with different exponential factors and hence related to an infinite number of relaxation times. However, if $\lambda_{-}=0$, which happens if the exchange integral $J=0$, the system reaches equilibrium already at the first step. If the $B / K$ ratio decreases, the relaxation process slows down and when $\lambda_{-} \rightarrow \lambda_{+}$the critical slowing down occurs. This phenomenon takes place irrespectively of the choice of the initial state, and the only necessary conditions of its occurrence are that $B \rightarrow 0$ and $K \rightarrow \infty$. The hitherto description was made with only one correlation function, magnetisation $\langle S\rangle$. In the next section we will show to which extent this description can be applied to the correlation function of neighbouring sites.

\section{Two-spin correlation function}

Up to now we have considered the cell with one spin and from now on we will assume the cell containing two spins. The expression of the transition matrix whose eigenvector contains the two-spin correlation function of neighbouring sites implies ambiguity in the matrix definition. Figure 2 presents two different possibilities of distribution of the three subsequent sites. In the first case the transition matrix takes the form

$$
\boldsymbol{m} \mathbf{1}=\left[\begin{array}{cccc}
\mathrm{e}^{2 K+2 B} & \mathrm{e}^{K+B} & \mathrm{e}^{-K+B} & 1 \\
\mathrm{e}^{-K+B} & \mathrm{e}^{-2 K} & 1 & \mathrm{e}^{K-B} \\
\mathrm{e}^{K+B} & 1 & \mathrm{e}^{-2 K} & \mathrm{e}^{-K-B} \\
1 & \mathrm{e}^{-K-B} & \mathrm{e}^{K-B} & \mathrm{e}^{2 K-2 B}
\end{array}\right]
$$

and in the second case

$$
\boldsymbol{m} \mathbf{2}=\left[\begin{array}{cccc}
\mathrm{e}^{2 K+2 B} & \mathrm{e}^{-K+B} & \mathrm{e}^{K+B} & 1 \\
\mathrm{e}^{K+B} & \mathrm{e}^{-2 K} & 1 & \mathrm{e}^{-K-B} \\
\mathrm{e}^{-K+B} & 1 & \mathrm{e}^{-2 K} & \mathrm{e}^{K-B} \\
1 & \mathrm{e}^{K-B} & \mathrm{e}^{-K-B} & \mathrm{e}^{2 K-2 B}
\end{array}\right]
$$
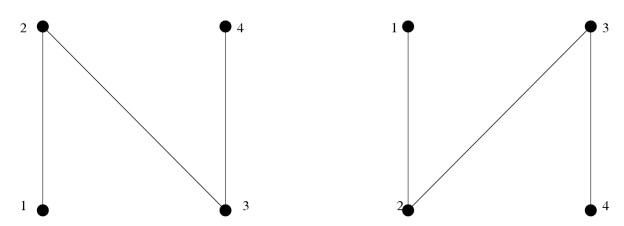

Fig. 2. Two methods of labelling sites at three subsequent bonds. The left hand side corresponds to $\boldsymbol{m} \mathbf{1}$ matrix, while the right hand side to $\mathbf{m 2}$ matrix.

The matrices $\boldsymbol{m} \mathbf{1}$ and $\boldsymbol{m} \mathbf{2}$ have two nonzero eigenvalues related to the eigenvalues of matrix $\boldsymbol{M}$ through the following expressions:

$$
\lambda_{1}=\lambda_{+}^{2}, \quad \lambda_{2}=\lambda_{-}^{2} .
$$

As the description of the relaxation in terms of matrix $\boldsymbol{m} \mathbf{1}$ or $\boldsymbol{m} \mathbf{2}$ leads to identical expressions for the physical quantities, we will consider only matrix $\boldsymbol{m} \mathbf{1}$. This matrix is asymmetrical so the right and left vectors corresponding to the same eigenvalue have different components, in contrast to the situation for matrix $\boldsymbol{M}$. Consequently, unlike the vectors $\left|\psi_{+}\right\rangle$and $\left|\psi_{-}\right\rangle$, the components of the eigenvectors do not have a simple physical interpretation. However, the probability can be still related to the products of the eigenvector coordinates.

Let the normalised to unity vectors $\left\langle\xi_{+}\right|$and $\left|\xi_{+}\right\rangle$correspond to the eigenvalue $\lambda_{1}$, while the vectors $\left\langle\xi_{-}\right|$and $\left|\xi_{-}\right\rangle$to the eigenvalue $\lambda_{2}$, then

$$
\begin{aligned}
& \left\langle\xi_{+}|[1] \cdot| \xi_{+}\right\rangle[1]=\left(1+\left\langle S_{1}\right\rangle+\left\langle S_{2}\right\rangle+\left\langle S_{1} S_{2}\right\rangle\right) / 4, \\
& \left\langle\xi_{+}|[2] \cdot| \xi_{+}\right\rangle[2]=\left(1+\left\langle S_{1}\right\rangle-\left\langle S_{2}\right\rangle-\left\langle S_{1} S_{2}\right\rangle\right) / 4, \\
& \left\langle\xi_{+}|[3] \cdot| \xi_{+}\right\rangle[3]=\left(1-\left\langle S_{1}\right\rangle+\left\langle S_{2}\right\rangle-\left\langle S_{1} S_{2}\right\rangle\right) / 4,
\end{aligned}
$$


$\left\langle\xi_{+}|[4] \cdot| \xi_{+}\right\rangle[4]=\left(1-\left\langle S_{1}\right\rangle-\left\langle S_{2}\right\rangle+\left\langle S_{1} S_{2}\right\rangle\right) / 4 . \quad(15)$ In these equations we have introduced the equilibrium magnetisation at site one and two $\left\langle S_{1}\right\rangle=\left\langle S_{2}\right\rangle=$ $\sinh B / \sqrt{\sinh ^{2} B+\mathrm{e}^{-4 K}}$ and the correlation function of the neighbouring spins $\left\langle S_{1} S_{2}\right\rangle=\left[\cosh B+\left(\sinh ^{2} B-\right.\right.$ $\left.\left.\mathrm{e}^{-4 K}\right) / \sqrt{\sinh ^{2} B+\mathrm{e}^{-4 K}}\right] /\left(\cosh B+\sqrt{\sinh ^{2} B+\mathrm{e}^{-4 K}}\right)$. The case of the eigenvectors corresponding to the lower eigenvalue $\lambda_{2}$ is more complex, as the free energy of an infinite system is expressed only in terms of $\lambda_{1}$, which permits expression of the products of the same vector coordinates through the correlation functions. For matrix $\boldsymbol{M}$, the components of the vector corresponding to the lower eigenvalue also had a simple physical interpretation and corresponded to the system with reversed spins. Looking for an analogy to the above we write the set of equations

$$
\begin{aligned}
& \left\langle\xi_{-}|[1] \cdot| \xi_{-}\right\rangle[1]=\left(1-\left\langle S_{1}\right\rangle_{I}-\left\langle S_{2}\right\rangle_{I}+\left\langle S_{1} S_{2}\right\rangle_{I}\right) / 4, \\
& \left\langle\xi_{-}|[2] \cdot| \xi_{-}\right\rangle[2]=\left(1-\left\langle S_{1}\right\rangle_{I}+\left\langle S_{2}\right\rangle_{I}-\left\langle S_{1} S_{2}\right\rangle_{I}\right) / 4, \\
& \left\langle\xi_{-}|[3] \cdot| \xi_{-}\right\rangle[3]=\left(1+\left\langle S_{1}\right\rangle_{I}-\left\langle S_{2}\right\rangle_{I}-\left\langle S_{1} S_{2}\right\rangle_{I}\right) / 4, \\
& \left\langle\xi_{-}|[4] \cdot| \xi_{-}\right\rangle[4]=\left(1+\left\langle S_{1}\right\rangle_{I}+\left\langle S_{2}\right\rangle_{I}+\left\langle S_{1} S_{2}\right\rangle_{I}\right) / 4,
\end{aligned}
$$

whose solutions are

$$
\left\langle S_{1}\right\rangle_{I}=\left\langle S_{2}\right\rangle_{I}=\langle S\rangle=\sinh B / \sqrt{\sinh ^{2} B+\mathrm{e}^{-4 K}}
$$

and

$\left\langle S_{1} S_{2}\right\rangle_{I}$

$$
=\frac{\mathrm{e}^{2 K}\left(\mathrm{e}^{2 K}+\mathrm{e}^{-2 K}\right)+2 \cosh B / \sqrt{\sinh ^{2} B+\mathrm{e}^{-4 K}}}{\mathrm{e}^{2 K}\left(\mathrm{e}^{2 K}+\mathrm{e}^{-2 K}\right)} .
$$

The new function defined in the above expressions $\left\langle S_{1} S_{2}\right\rangle_{I}$ behaves similarly as the inverse correlation function $\left\langle S_{1} S_{2}\right\rangle^{-1}$. In particular, for $B=0\left\langle S_{1} S_{2}\right\rangle_{I}=$ $(\tanh K)^{-1}=\left\langle S_{1} S_{2}\right\rangle^{-1}$.

If the cell transformed by matrix $\boldsymbol{m} \mathbf{1}$ is not a single spin but a pair of spins, the non-equilibrium state of this pair $|\phi\rangle$, related to matrix $\boldsymbol{M}$, must be replaced by the state $|\mu\rangle$, defined by the equation:

$$
|\mu\rangle=\left\langle\xi_{+} \mid \mu\right\rangle\left|\xi_{+}\right\rangle+\left\langle\xi_{-} \mid \mu\right\rangle\left|\xi_{-}\right\rangle .
$$

In analogy to the state $\left|\phi_{n}\right\rangle$, we find the state $\left|\mu_{n}\right\rangle$ :

$$
\begin{aligned}
& \left|\mu_{n}\right\rangle= \\
& \frac{\lambda_{1}^{n}\left\langle\xi_{+} \mid \mu_{0}\right\rangle\left|\xi_{+}\right\rangle+\lambda_{2}^{n}\left\langle\xi_{-} \mid \mu_{0}\right\rangle\left|\xi_{-}\right\rangle}{\sqrt{\lambda_{1}^{2 n}\left\langle\xi_{+} \mid \mu_{0}\right\rangle\left\langle\mu_{0} \mid \xi_{+}\right\rangle+\lambda_{2}^{2 n}\left\langle\xi_{-} \mid \mu_{0}\right\rangle\left\langle\mu_{0} \mid \xi_{-}\right\rangle}},
\end{aligned}
$$

where $\left|\mu_{0}\right\rangle$ is the initial state. The probabilities describing the two spin states at $t=n$ is found from subsequent products of the coordinates of the vector $\left|\mu_{n}\right\rangle$ :

$$
\begin{aligned}
& P_{n}(\uparrow \uparrow)=\left\langle\mu_{n}|[1] \cdot| \mu_{n}\right\rangle[1]=\left(1+\left\langle S_{1}\right\rangle_{n}+\left\langle S_{2}\right\rangle_{n}\right. \\
& \left.\quad+\left\langle S_{1} S_{2}\right\rangle_{n}\right) / 4 \\
& \quad=\frac{1}{D}\left\{\lambda_{1}^{2 n}\left\langle\xi_{+} \mid \mu_{0}\right\rangle\left\langle\mu_{0} \mid \xi_{+}\right\rangle\left\langle\xi_{+}|[1] \cdot| \xi_{+}\right\rangle[1]\right.
\end{aligned}
$$

$$
\begin{aligned}
& +\lambda_{2}^{2 n}\left\langle\xi_{-} \mid \mu_{0}\right\rangle\left\langle\mu_{0} \mid \xi_{-}\right\rangle\left\langle\xi_{-}|[1] \cdot| \xi_{-}\right\rangle[1] \\
& +\lambda_{1}^{n} \lambda_{2}^{n}\left[\left\langle\mu_{0} \mid \xi_{+}\right\rangle\left\langle\xi_{-} \mid \mu_{0}\right\rangle\left\langle\xi_{+}|[1] \cdot| \xi_{-}\right\rangle[1]\right. \\
& \left.\left.+\left\langle\mu_{0} \mid \xi_{-}\right\rangle\left\langle\xi_{+} \mid \mu_{0}\right\rangle\left\langle\xi_{-}|[1] \cdot| \xi_{+}\right\rangle[1]\right]\right\}, \\
& P_{n}(\uparrow \downarrow)=\left\langle\mu_{n}|[2] \cdot| \mu_{n}\right\rangle[2]=\left(1+\left\langle S_{1}\right\rangle_{n}-\left\langle S_{2}\right\rangle_{n}\right. \\
& \left.-\left\langle S_{1} S_{2}\right\rangle_{n}\right) / 4 \\
& =\frac{1}{D}\left\{\lambda_{1}^{2 n}\left\langle\xi_{+} \mid \mu_{0}\right\rangle\left\langle\mu_{0} \mid \xi_{+}\right\rangle\left\langle\xi_{+}|[2] \cdot| \xi_{+}\right\rangle[2]\right. \\
& +\lambda_{2}^{2 n}\left\langle\xi_{-} \mid \mu_{0}\right\rangle\left\langle\mu_{0} \mid \xi_{-}\right\rangle\left\langle\xi_{-}|[2] \cdot| \xi_{-}\right\rangle[2] \\
& +\lambda_{1}^{n} \lambda_{2}^{n}\left[\left\langle\mu_{0} \mid \xi_{+}\right\rangle\left\langle\xi_{-} \mid \mu_{0}\right\rangle\left\langle\xi_{+}|[2] \cdot| \xi_{-}\right\rangle[2]\right. \\
& \left.\left.+\left\langle\mu_{0} \mid \xi_{-}\right\rangle\left\langle\xi_{+} \mid \mu_{0}\right\rangle\left\langle\xi_{-}|[2] \cdot| \xi_{+}\right\rangle[2]\right]\right\}, \\
& P_{n}(\downarrow \uparrow)=\left\langle\mu_{n}|[3] \cdot| \mu_{n}\right\rangle[3]=\left(1-\left\langle S_{1}\right\rangle_{n}+\left\langle S_{2}\right\rangle_{n}\right. \\
& \left.-\left\langle S_{1} S_{2}\right\rangle_{n}\right) / 4 \\
& =\frac{1}{D}\left\{\lambda_{1}^{2 n}\left\langle\xi_{+} \mid \mu_{0}\right\rangle\left\langle\mu_{0} \mid \xi_{+}\right\rangle\left\langle\xi_{+}|[3] \cdot| \xi_{+}\right\rangle[3]\right. \\
& +\lambda_{2}^{2 n}\left\langle\xi_{-} \mid \mu_{0}\right\rangle\left\langle\mu_{0} \mid \xi_{-}\right\rangle\left\langle\xi_{-}|[3] \cdot| \xi_{-}\right\rangle[3] \\
& +\lambda_{1}^{n} \lambda_{2}^{n}\left[\left\langle\mu_{0} \mid \xi_{+}\right\rangle\left\langle\xi_{-} \mid \mu_{0}\right\rangle\left\langle\xi_{+}|[3] \cdot| \xi_{-}\right\rangle[3]\right. \\
& \left.\left.+\left\langle\mu_{0} \mid \xi_{-}\right\rangle\left\langle\xi_{+} \mid \mu_{0}\right\rangle\left\langle\xi_{-}|[3] \cdot| \xi_{+}\right\rangle[3]\right]\right\}, \\
& P_{n}(\downarrow \downarrow)=\left\langle\mu_{n}|[4] \cdot| \mu_{n}\right\rangle[4]=\left(1-\left\langle S_{1}\right\rangle_{n}-\left\langle S_{2}\right\rangle_{n}\right. \\
& \left.+\left\langle S_{1} S_{2}\right\rangle_{n}\right) / 4 \\
& =\frac{1}{D}\left\{\lambda_{1}^{2 n}\left\langle\xi_{+} \mid \mu_{0}\right\rangle\left\langle\mu_{0} \mid \xi_{+}\right\rangle\left\langle\xi_{+}|[4] \cdot| \xi_{+}\right\rangle[4]\right. \\
& +\lambda_{2}^{2 n}\left\langle\xi_{-} \mid \mu_{0}\right\rangle\left\langle\mu_{0} \mid \xi_{-}\right\rangle\left\langle\xi_{-}|[4] \cdot| \xi_{-}\right\rangle[4] \\
& +\lambda_{1}^{n} \lambda_{2}^{n}\left[\left\langle\mu_{0} \mid \xi_{+}\right\rangle\left\langle\xi_{-} \mid \mu_{0}\right\rangle\left\langle\xi_{+}|[4] \cdot| \xi_{-}\right\rangle[4]\right. \\
& \left.\left.+\left\langle\mu_{0} \mid \xi_{-}\right\rangle\left\langle\xi_{+} \mid \mu_{0}\right\rangle\left\langle\xi_{-}|[4] \cdot| \xi_{+}\right\rangle[4]\right]\right\},
\end{aligned}
$$
where

$$
D=\lambda_{1}^{2 n}\left\langle\xi_{+} \mid \mu_{0}\right\rangle\left\langle\mu_{0} \mid \xi_{+}\right\rangle+\lambda_{2}^{2 n}\left\langle\xi_{-} \mid \mu_{0}\right\rangle\left\langle\mu_{0} \mid \xi_{-}\right\rangle .
$$

These probabilities permit determination of the spin correlation functions in the cell at time $t=n$ :

$$
\begin{aligned}
& \left\langle S_{1}\right\rangle_{n}=P_{n}(\uparrow \uparrow)+P_{n}(\uparrow \downarrow)-P_{n}(\downarrow \uparrow)-P_{n}(\downarrow \downarrow), \\
& \left\langle S_{2}\right\rangle_{n}=P_{n}(\uparrow \uparrow)-P_{n}(\uparrow \downarrow)+P_{n}(\downarrow \uparrow)-P_{n}(\downarrow \downarrow), \\
& \left\langle S_{1} S_{2}\right\rangle_{n}=P_{n}(\uparrow \uparrow)-P_{n}(\uparrow \downarrow)-P_{n}(\downarrow \uparrow)+P_{n}(\downarrow \downarrow) .
\end{aligned}
$$

These expressions are much more complicated than those corresponding to the cell with a single site. Moreover, for the cell with two sites the conditions specifying the initial state are different. For the cell with a single site the initial state was fully described by the values of the magnetic moments in each cell. For the system made of the cells with two sites we can expect that not only the 
values of $\left\langle S_{1}\right\rangle_{0},\left\langle S_{2}\right\rangle_{0}$ and $\left\langle S_{1} S_{2}\right\rangle_{0}$ but also $\left(\left\langle S_{1} S_{2}\right\rangle_{I}\right)_{0}$ should be specified. However, the situation is even more complicated than that. As matrix $\boldsymbol{m} \mathbf{1}$ is asymmetrical we do not know the functional expressions for the components $\left|\xi_{+}\right\rangle$and $\left|\xi_{-}\right\rangle$so also for $\left|\mu_{0}\right\rangle$. Therefore, to be able to apply the equations derived we have to assume that the initial vector $\left|\mu_{0}\right\rangle$ is the eigenvector of matrix $\boldsymbol{m} \mathbf{1}$, although it corresponds to different values of $B$ and $K$.

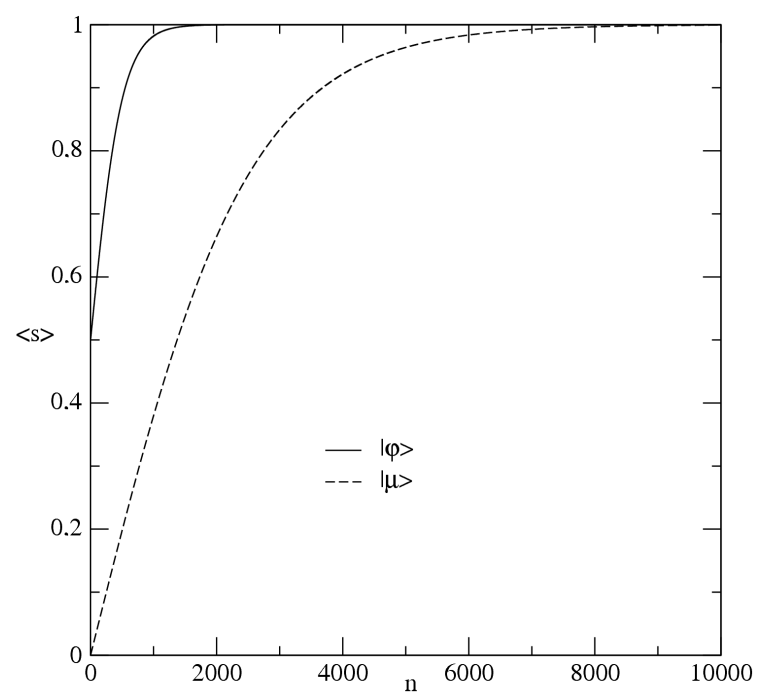

Fig. 3. Time dependence of magnetisation when the equilibrium conditions are specified by $K=10$ and $B=$ 0.0001 for the single-site cell — solid line, and for the two-site cells - broken line. The initial state of the single-site cell is that with $\langle S\rangle$, while that of the two-site cell is the equilibrium state at $K=0.1$ and $B=0.0001$.
The magnetisation at $K=10$ and $B=0.0001$, for the initial state with $K=0.1$ and $B=0.0001$ was calculated and the result was compared with the magnetisation values of the cell with one site undergoing evolution in the same conditions $K=10, B=0.0001$ and for zero initial magnetisation value. As follows from Fig. 3, in the system with two-spin cells the process of relaxation is much slower than in the system of the cells with one site. The first factor responsible for the slowing down is the twice longer characteristic time defined by $\lambda_{2} / \lambda_{1}$. Moreover, for the system with two-site cells the relaxation of magnetisation is accompanied by the process of reaching equilibrium by the correlation function $\left\langle S_{1} S_{2}\right\rangle$, which does not take place in the system of single-site cells. For a two-side cell, the critical slowing down is observed when $\lambda_{1}$ approaches $\lambda_{2}$, for both one-spin and two-spin correlation functions.

\section{References}

[1] K. Kawasaki, in: Phase Transitions and Critical Phenomena, Eds. C. Domb, M.S. Green, Vol. 2, Academic, New York 1972, p. 443.

[2] S. Wolfram, Rev. Mod. Phys. 55, 601 (1983).

Eds. C. Domb, M.S. Green, Vol. 2, Academic, New York 1972 .

[3] E. Domany, W. Kinzel, Phys. Rev. Lett. 53, 311 (1984).

[4] L.E. Reichl, A Modern Course in Statistical Physics, Edward Arnold Ltd, University of Texas Press, Austin 1980, p. 193.

[5] M. Błaszyk, Acta Phys. Pol. A 107, 451 (2005). 